\title{
Validación de un método analítico para la determinación del contenido de sodio en los alimentos
}

Ericka Valverde Montero'

Paulina Silva Trejos ${ }^{2}$

Fecha de recepción: 25 de setiembre del 2011

Fecha de aceptación: 20 de enero del 2012

Valverde, E; Silva, P. Validación de un método analítico para la determinación del contenido de sodio en los alimentos. Tecnología en Marcha. Vol. 25, Nº 2. Abril-Junio 20 I2. Pág 4I-49.

Química. Escuela de Seguridad laboral e Higiene Ambiental. Tecnológico de Costa Rica. Teléfono: 25II-4870 Correo electrónico: erickavm@gmail.com

2 Química. Escuela de Química, Universidad de Costa Rica. Teléfono: 25 I I-4870 Correo electrónico: stpaulinita@gmail.com 


\section{Resumen}

Se validó la metodología analítica para la determinación de sodio en alimentos mediante espectroscopía de absorción atómica de llama. Las muestras de $0,5 \mathrm{~g}$ se digirieron en un horno de microondas con 5,0 $\mathrm{mL}$ de $\mathrm{HNO}_{3}$ al $65 \%$ en masa. El ámbito de linealidad óptimo que se obtuvo fue de $0,043 \mathrm{mg} / \mathrm{L}$ a $0,70 \mathrm{mg} / \mathrm{L}$ con un coeficiente de correlación de 0,998.

Los límites de detección y cuantificación reportados fueron de 0,025 mg/L y 0,043 mg/L, respectivamente; con una sensibilidad de calibración de 0,805 $\mathrm{Lmg}^{-1}$, una sensibilidad analítica de $44 \mathrm{Lmg}^{-1}$. La precisión se evaluó en condiciones de repetibilidad y se obtuvo un valor para RDS, igual a 2,9\%. La veracidad se determinó utilizando tres patrones certificados del NIST@, SRM 1846 Infant Formula con un valor reportado para sodio de $(2310 \pm 130) \mathrm{mg} / \mathrm{kg}$, SRM 84 I 4 Bovine Muscle Powder con $(0,210 \pm 0,008) \%$ en masa, y SRM 84I 5 Whole Egg Powder con $(0,377$ $\pm 0,034) \%$ en masa.

Se obtuvieron sesgos de entre $(-0,010$ a 0,009$)$ $\mathrm{mg} / \mathrm{L}$. De la lista de alimentos seleccionados para el estudio, se encontró que la leche entera en polvo, el pan blanco de trigo, el queso fresco y el queso mozzarella presentan el contenido más elevado en sodio, con concentraciones que oscilan entre (I06 a 452) $\mathrm{mg} \mathrm{Na} / \mathrm{l00g}$.

\section{Palabras clave}

Sodio, macro mineral, nutriente, alimentos, sal, hipertensión.

\section{Abstract}

The analytical methodology for quantitative determination of sodium in foods by flame atomic absorption spectrometry was validated. The sample digestion of $0,5 \mathrm{~g}$ was realized by microwave oven with $5,0 \mathrm{~mL}$ of $65 \%$ nitric acid. The linearity range was from $0,043 \mathrm{mg} / \mathrm{L}$ to $0,70 \mathrm{mg} / \mathrm{L}$ with a correlation coefficient equal to 0,998.

The detection and quantification limits were 0,025 $\mathrm{mg} / \mathrm{L}$ and 0,043 mg/L, respectively. Whit 0,805 Lmg ' of calibration sensitivity and $44 \mathrm{Lmg}^{-1}$ of analytical sensitivity. The precision was evaluated in terms of repeatability, we obtained a value equal to 2,9\% RDSr. The trueness was determined using three NIST\& certified standards SRM 1846 Infant Formula with a reported value sodium of $(2310 \pm 130) \mathrm{mg} / \mathrm{kg}$, SRM I 846 Bovine Muscle Powder with a reported value for sodium of $(0.210 \pm 0.008) \%$ and SRM 8415 Whole Egg Powder with a reported value for sodium of $0,377 \pm 0,034) \%$ by mass.

We obtained an average bias between $(-0,010$ to $0,009) \mathrm{mg} / \mathrm{L}$. From the list of foods selected for the study, we found that whole milk powder, white wheat bread, fresh cheese and mozzarella cheese have the highest content in sodium concentrations, ranging from (I06 to 452) $\mathrm{mg} \mathrm{Na} \mathrm{//00g}$

\section{Key words}

Sodium, macro mineral, nutrient, foods, salt, hypertension. 


\section{Introducción}

El sodio es el catión más abundante del organismo; el $70 \%$ del sodio corporal total existe en forma libre, de este, el 97\% se encuentra en el líquido extracelular y el 3\% en el líquido intracelular; el 30\% restante está en forma fija, no intercambiable en el hueso, cartílago y tejido conectivo. La concentración sérica de sodio es mantenida por mecanismos que involucran la sed, la hormona antidiurética y la función renal. ${ }^{1,2}$

La cantidad de sodio requerida para un adulto se estima en unos $500 \mathrm{~g} /$ día. Sin embargo, la ingesta normal de sodio en forma de cloruro de sodio varía de (2-14) g por día.,

Existe evidencia científicamente comprobada de que el excesivo consumo de sal genera hipertensión arterial HTA y que esta a su vez es un factor de riesgo para las enfermedades cardiovasculares (ECV). 4,5,6,7,

Actualmente se desconoce la ingesta real de sodio de la población costarricense. La Encuesta Nacional sobre Consumo de Sal Doméstica en el Hogar realizada en 1984 indicó un consumo promedio de $\mathrm{NaCl}$ de $(\mid$ 0,6-|3,5) g/día, equivalente a (4,2-5,3) $\mathrm{g} \mathrm{Na/día.} \mathrm{En} \mathrm{esta} \mathrm{encuesta} \mathrm{se} \mathrm{identificaron} \mathrm{algunos}$ alimentos que aportan cantidades significativas de sodio en la dieta: el pan "francés", el arroz, el queso fresco, la margarina y el pan dulce. Se estimó que el consumo de alimentos procesados, sopas deshidratadas, consomés, salsas y embutidos contribuye altamente a la ingesta elevada de este mineral., 10

Una encuesta realizada en Cartago en el 200 I mostró un consumo per cápita promedio de $3661 \mathrm{mg} \mathrm{Na} /$ día. Se identificaron como las fuentes principales la sal doméstica con $66 \%$, el pan y las galletas con I2\%, el consomé con $7 \%$ y los embutidos con $6 \%$." En este caso, el consumo disminuyó, sin embargo, aún sobrepasa el valor indicado por la Organización Mundial de la Salud (OMS) de I 700 mg Na / día, entre sus recomendaciones para reducir la presión arterial. ${ }^{12}$

El exceso de cloruro de sodio aumenta la cantidad de agua fuera de la célula, lo que provoca retención de líquido y aumenta la presión de la sangre. Hay evidencias de que una alimentación baja en sodio reduce la presión arterial en personas hipertensas y disminuye la necesidad de medicamentos antihipertensivos. ${ }^{\prime \prime}$
En Costa Rica, el 25\% de la población mayor de 18 años es hipertensa y el 55\% desconoce que la hipertensión genera enfermedad cardiovascular.13 Además, la hipertensión es uno de los trastornos crónicos con mayores costos de consulta externa. En el 2006, un trabajo presentado por la Asociación Costarricense de Salud Pública indicó una prevalencia de HTA en el $59 \%$ de los adultos mayores. ${ }^{14}$

En el 2005 se realizó un estudio sobre la calidad de atención en hipertensión arterial, el cual demostró que al 29\% de las personas hipertensas estudiadas ( 1 59 I) se le diagnosticó daño a órgano blanco o enfermedad cardiovascular. Asimismo, se detectó un aumento del número de personas hipertensas conforme aumenta la edad. ${ }^{15}$

En un informe actuarial sobre atención de enfermedades, la Caja Costarricense de Seguro Social (CCSS) indicó que solo en el 2009 los daños en el sistema circulatorio (de los cuales el $80 \%$ son infartos, derrames cerebrales e hipertensión arterial) ocasionaron una tasa de mortalidad de I I 5 por cada 100000 habitantes. ${ }^{16}$

En una evaluación de las principales fuentes alimentarias que contribuyen a la ingesta de sodio en los hogares británicos, elaborada por The National Food Survey, se encontró que, excluyendo la sal añadida en la preparación de los alimentos y el sodio consumido fuera de casa, los cereales y productos de cereales (que incluyen pan, cereales para el desayuno, galletas y pasteles) proporcionan cerca del $40 \%$ de la ingesta media de sodio, y la carne y los productos cárnicos aportan aproximadamente el $21 \%{ }^{17}$

A nivel mundial ya se han tomado medidas para prevenir los daños causados por el consumo excesivo de sodio y sal. En el 2005 se estableció el grupo Acción Mundial sobre la Sal y la Salud (WASH), con la misión de mejorar es lograr una reducción en la ingesta de sodio en todo el mundo mediante la reducción de la cantidad de sal en los alimentos procesados, así como la sal de mesa y la empleada en la cocina. ${ }^{18}$

En el 2009, la Organización Panamericana de la Salud y la OMS impulsaron la iniciativa sobre la reducción del consumo de sal en el continente americano, con el propósito de prevenir las enfermedades cardiovasculares. Uno de los objetivos principales es 


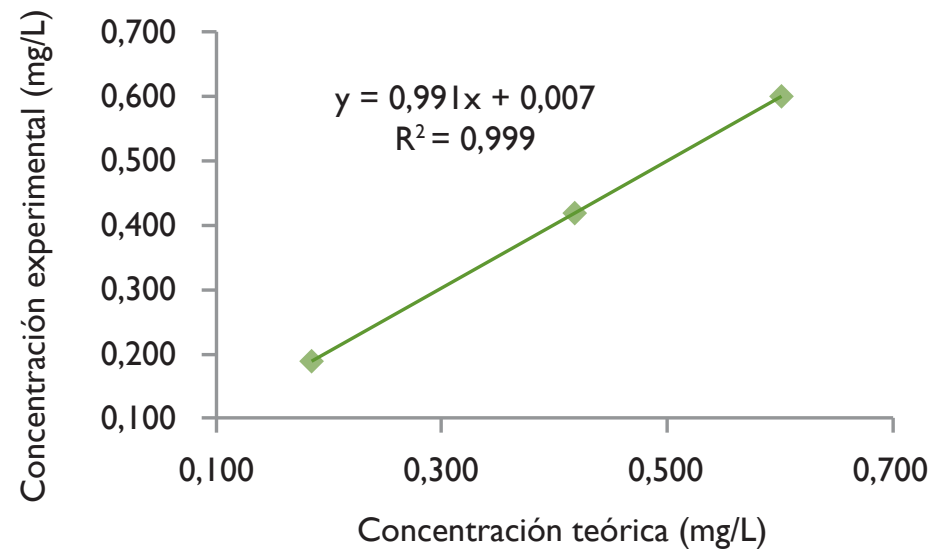

Figura I. Estudio de veracidad para sodio en MRC 84 I4.

revisar las políticas regionales y programas existentes y emitir recomendaciones para reducir gradualmente el consumo de sal en la dieta, con el fin de alcanzar las metas nacionales e internacionalmente recomendadas, de mantener un valor inferior a $5 \mathrm{~g}$ por persona al día para el 2020. ${ }^{19}$

Debido a la necesidad de investigar el contenido de sodio en la dieta, en este trabajo se validó un método analítico para determinar el contenido de sodio en diferentes grupos de alimentos de importante consumo a nivel nacional. Los resultados generados proporcionan datos directos y primarios para las bases de datos de composición de alimentos, que son necesarias en la evaluación de los aportes nutricionales y determinar la idoneidad de su consumo por parte de la población.

\section{Metodología}

\section{Preparación de las muestras}

Las muestras analizadas en este estudio fueron recolectadas cada cuatro meses en diferentes puntos de venta aledaños a la Ciudad Universitaria Rodrigo Facio en San Pedro de Montes de Oca, durante un periodo de un año. Para la preparación de las muestras se tomaron como base los lineamientos indicados por Greenfield y Southgate. ${ }^{20}$

Se analizaron 46 tipos diferentes de alimentos que incluyen seis grupos: cereales y derivados, carnes y derivados, lácteos, verduras y hortalizas, leguminosas y el grupo de las frutas. El proceso para cada alimento dependió del tipo. Se prepararon según el consumo más habitual, ya sean frescos o cocidos, sin agregarles aditivos. El tamaño de cada muestra dependió del tamaño de las unidades individuales del alimento con el fin de que, en total, la muestra primaria tuviera una masa de $(0,1-0,5) \mathrm{kg}$.

En cada muestreo se tomaron tres muestras primarias, a partir de las cuales se formó una muestra compuesta de $(0,1-0,2) \mathrm{kg}$ con la parte comestible del alimento. Se tomó una pequeña porción para la determinación de la humedad y el resto se liofilizó y se pesó con exactitud para su posterior digestión. Las muestras de 0,5 g se digirieron en un horno de microondas con 5,0 $\mathrm{mL}$ de $\mathrm{HNO}_{3}$ al $65 \%$ en masa, calidad A.C.S. El programa de digestión se aplicó en tres etapas, con tiempo y potencia establecidos de acuerdo con la matriz y el manual del equipo. ${ }^{21}$

\section{Procedimiento analítico}

Después de la digestión por duplicado del alimento liofilizado, las submuestras se diluyeron en balones aforados de $50 \mathrm{~mL}$ con agua desionizada y se trasvasaron a recipientes de Nalgene.La dilución de las muestras se realizó con $\mathrm{KCl}(2000 \mu \mathrm{g} / \mathrm{mL})$ y $\mathrm{HNO}_{3}$ al $1 \%$ y las mediciones se realizaron con un espectrofotómetro de absorción atómica Varian SpectrAA, modelo 220 Fast Sequential, provisto de una lámpara de sodio y una llama aire/ $\mathrm{C}_{2} \mathrm{H}_{2}$. Se trabajó a una longitud de onda igual a $589 \mathrm{~nm}$. Las curvas de calibración se prepararon utilizando una disolución estándar de sodio JT. Baker N 6468-04 1000 trazable al NIST®, con una concentración de ( $1000 \pm \mathrm{I}) \mu \mathrm{g} / \mathrm{mL}$ al $5 \%$ en $\mathrm{HNO}_{3}$.

Se empleó como base el Método Oficial 985.35 del $\mathrm{AOAC}^{22}$ para el desarrollo del método, con algunas 
Cuadro I. Resultados de desviación estándar relativa para la determinación de sodio en tres niveles de concentración.

\begin{tabular}{|c|c|c|c|}
\hline Sodio & Nivel I & Nivel 2 & Nivel 3 \\
\hline MRCI846 & 3,6 & 2,8 & 2,6 \\
\hline MRC 84I4 & 3,8 & 2,8 & 2,5 \\
\hline MRC 84I5 & 3,3 & 3,0 & 3,0 \\
\hline RSDr promedio & 3,6 & 2,9 & 2,6 \\
\hline
\end{tabular}

modificaciones tales como: la ampliación a diferentes matrices, digestión con horno de microondas y sustitución de $\mathrm{CsCl}$ empleado como supresor de ionización por $\mathrm{KCl}$. Los porcentajes de humedad se determinaron por duplicado para cada alimento fresco o cocido según el caso, siguiendo los métodos oficiales del AOAC.

\section{Resultados}

Para evaluar el comportamiento de la curva de calibración se realizaron pruebas estadísticas de homogeneidad: la prueba de Cochran y la prueba de Bartlett; ambas indicaron un comportamiento homocedástico, para una probabilidad de 0,05. El ámbito de linealidad óptimo fue de (0,043-0,70) $\mathrm{mg} / \mathrm{L}$ con un coeficiente de correlación igual a 0,998.

La sensibilidad de calibración mide la capacidad del método para discernir pequeñas variaciones en la concentración. Por cada mg/L de analito, se produce un incremento en unidades de absorbancia equivalente a la pendiente de la curva de calibración.
La sensibilidad de calibración se obtuvo a partir del promedio de las pendientes de varias curvas de calibración y fue de 0,805 A. $\mathrm{Lmg}^{-1}$ y la sensibilidad analítica fue de $44 \mathrm{Lmg}^{-1}$. Este valor se obtiene al dividir la pendiente entre la desviación estándar de la señal analítica en unidades de absorbancia.

Los límites de detección y de cuantificación se determinaron mediante el método gráfico descrito por Meier y Zünd, ${ }^{23}$ el cual utiliza la curva de calibración y los límites de confianza al 95\%. Los resultados obtenidos fueron de 0,025 mg/L y 0,043 $\mathrm{mg} / \mathrm{L}$, respectivamente.

La ausencia de errores sistemáticos para los tres materiales de referencia se demuestra mediante la comparación gráfica entre los valores experimentales y los valores teóricos (figura I). Para todos los elementos en estudio, el análisis de la recta de regresión al 95\% de confianza demostró un coeficiente de correlación no significativamente diferente de uno, pendiente no significativamente diferente de uno y ordenada en el origen no significativamente diferente de cero.

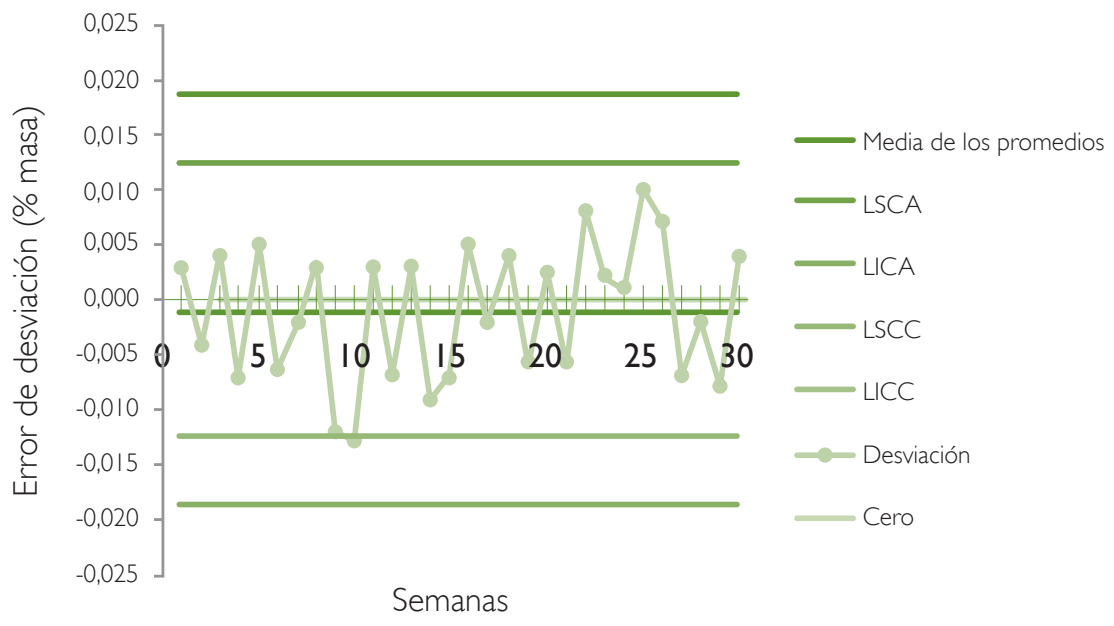

Figura 2. Estudio de estabilidad para sodio en MRC 84I4 Bovine Muscle Powder. 
La precisión se evaluó en condiciones de repetibilidad; se determinó para los materiales de referencia SRM I846 Infant Formula, SRM 1846 Bovine Muscle Powder y SRM 84I5 Whole Egg Powder, en tres niveles de concentración. Los resultados se observan en el cuadro. La veracidad se evaluó para los mismos materiales de referencia, SRM 1846 Infant Formula con un valor reportado para sodio de (2310 130) $\mathrm{mg} / \mathrm{kg}$, SRM 1846 Bovine Muscle Powder con $(0,210 \pm 0,008) \%$ en masa y SRM 8415 Whole Egg Powder $(0,377 \pm 0,034) \%$ en masa, en los tres casos se obtuvieron sesgos entre $(-0,010$ a 0,009$) \mathrm{mg} / \mathrm{L}$ con un nivel de confianza del $95 \%$.

Las etapas de digestión y medición de las muestras de alimentos seleccionados se llevaron a cabo bajo un sistema de control de calidad. Cada cuatro muestras se analizó un ensayo en blanco; cada 10 muestras se comprobó la veracidad al realizar una medición de una muestra de material de referencia certificado, y cada corrida de lectura se verificó la respuesta del espectrómetro de absorción atómica con una disolución patrón de sodio de concentración intermedia trazable al NIST®.

Para evaluar la estabilidad del método de medición, se empleó un gráfico de estabilidad (figura 2), en el cual se observa el error de desviación en \% en masa con respecto al valor teórico indicado en el material de referencia MRC 8414 Bovine Muscle Powder. Las mediciones se realizaron una vez por semana durante seis meses. Cuando las oscilaciones aleatorias de los datos en el tiempo alrededor de un valor central fijo se distribuyen normalmente, es indicativo de que el sistema se encuentra bajo control estadístico.

En los cuadros 2 a 7 se muestran los resultados de humedad y el contenido de sodio, expresado por porción de $100 \mathrm{~g}$ de alimento fresco para cada grupo de alimentos.

Los alimentos estudiados que presentan mayor contenido en sodio son los alimentos procesados, como el caso de la leche en polvo (452 mg Na/l00 g), que equivale en su forma reconstituida a (I 45 mg Na/l 00 g), el pan de trigo (430 mg Na/l 00 g), la tortilla (90 mg Na/l00 g), el queso crema (425 mg $\mathrm{Na} / \mathrm{l} 00 \mathrm{~g}$ ), el queso mozzarella (250 mg Na/l00 g) y el queso fresco ( $106 \mathrm{mg} \mathrm{Na/l00} \mathrm{g).}$

El contenido de sodio es muy variable entre los quesos, debido a las diferencia de humedad y al proceso de elaboración de los mismos, en el cual se agrega $\mathrm{NaCl}$ para diversos propósitos. Las carnes presentaron menor cantidad, entre (44-70) mg $\mathrm{Na} / \mathrm{OO0}$ g, al igual que el yogurt (53-80) mg Na/I00 g y la leche fluida descremada con $46 \mathrm{mg} \mathrm{Na/l} 00 \mathrm{~g}$. Los alimentos con menor contenido de sodio son las verduras (2-24) $\mathrm{mg} \mathrm{Na} / \mathrm{l} 00 \mathrm{~g}$ y las frutas, con menos de $2 \mathrm{mg} \mathrm{Na} / \mathrm{l} 00 \mathrm{~g}$.

Cuadro 2. Contenido de humedad y de sodio en lácteos y derivados.

\begin{tabular}{|l|c|c|}
\hline \multicolumn{1}{|c|}{ Alimento } & \% humedad & $\begin{array}{c}\text { Na mg /I00 g de } \\
\text { alimento fresco }\end{array}$ \\
\hline $\begin{array}{l}\text { Leche entera en polvo reconstituida, instantánea, I00\% vaca, } \\
\text { enriquecida con vitaminas A, D, ácido fólico y Fe }\end{array}$ & n.a & $145 \pm 5$ \\
\hline $\begin{array}{l}\text { Leche entera en polvo, instantánea, I00\% vaca, } \\
\text { enriquecida con vitaminas A, D, ácido fólico y Fe }\end{array}$ & n.a & $452 \pm$ I6 \\
\hline $\begin{array}{l}\text { Leche fluida descremada, I00\% vaca, enriquecida con vitaminas A, C, } \\
\text { D, ácido fólico y Fe }\end{array}$ & $66 \pm 2$ \\
\hline Queso crema, tipo americano, pasteurizado & 62,4 & $424 \pm 15$ \\
\hline Queso fresco pasteurizado, nacional & 55,0 & $106 \pm 4$ \\
\hline Queso mozzarella, pasteurizado nacional & n.a & $250 \pm 9$ \\
\hline Yogurt pasteurizado, azucarado, aromatizado, con albaricoque & n.a & $64 \pm 2$ \\
\hline Yogurt fluido pasteurizado, azucarado, aromatizado, sabor a fresa & n.a & $80 \pm 3$ \\
\hline $\begin{array}{l}\text { Yogurt semidescremado, pasteurizado, con cultivos probióticos, } \\
\text { azucarado, aromatizado, sabor a fresa }\end{array}$ & & $46 \pm 2$ \\
\hline
\end{tabular}


Cuadro 3. Contenido de humedad y de sodio en cereales y derivados.

\begin{tabular}{|c|c|c|}
\hline Alimento & \% humedad & $\begin{array}{c}\text { Na mg / } 00 \text { g de } \\
\text { alimento fresco }\end{array}$ \\
\hline $\begin{array}{c}\text { Arroz precocido, grano entero, cocido con agua desionizada en horno } \\
\text { de microondas, enriquecido con niacina, vitamina BI, vitamina B I2, } \\
\text { ácido fólico, zinc y selenio }\end{array}$ & 51,30 & $7,2 \pm 0,3$ \\
\hline $\begin{array}{c}\text { Arroz pulido, grano entero, cocido con agua desionizada en horno de } \\
\text { microondas, enriquecido con niacina, vitamina BI, vitamina B I, ácido } \\
\text { fólico, zinc y selenio }\end{array}$ & 51,95 & $4,9 \pm 0,2$ \\
\hline $\begin{array}{c}\text { Avena en hojuelas, cocida con agua desionizada en microondas, } \\
\text { nacional }\end{array}$ & 68,0 & $0,30 \pm 0,1$ \\
\hline Pan, trigo, cuadrado, blanco, nacional & 38,0 & $430 \pm 15$ \\
\hline $\begin{array}{c}\text { Pasta, caracolitos, pasta al huevo, cocida en agua desionizada sin aditivos } \\
\text { en horno de microondas }\end{array}$ & 66,5 & $\mathrm{ND}$ \\
\hline $\begin{array}{c}\text { Pasta, tornillos, pasta al huevo, cocida en agua desionizada sin aditivos } \\
\text { en horno de microondas }\end{array}$ & 58,0 & $90 \pm 3$ \\
\hline Tortillas, maíz, blanco tratado con cal, cocida industrial, nacional & 49,0 & $\mathrm{ND}$ \\
\hline
\end{tabular}

Cuadro 4. Contenido de humedad y de sodio en leguminosas y derivados.

\begin{tabular}{|l|c|c|}
\hline \multicolumn{1}{|c|}{ Alimento } & \% humedad & $\begin{array}{c}\text { Na mg } / \mathrm{l00} \text { g de } \\
\text { alimento fresco }\end{array}$ \\
\hline $\begin{array}{l}\text { Frijol negro, semilla entera, cocido en agua desionizada en horno de } \\
\text { microondas, nacional }\end{array}$ & 80,35 & $12,0 \pm 0,4$ \\
\hline $\begin{array}{l}\text { Frijol rojo, semilla entera, cocido en agua desionizada en horno de } \\
\text { microondas, nacional }\end{array}$ & 71,6 & $12,7 \pm 0,1$ \\
\hline $\begin{array}{l}\text { Garbanzos cocidos, semilla entera, cocidos en agua desionizada en } \\
\text { horno de microondas, nacional }\end{array}$ & 68,8 & $20,0 \pm 0,7$ \\
\hline $\begin{array}{l}\text { Lentejas, semilla entera, cocidas en agua desionizada en horno de } \\
\text { microondas, nacional }\end{array}$ & 73,23 & $17,4 \pm 0,6$ \\
\hline
\end{tabular}

Cuadro 5. Contenido de humedad y de sodio en carnes y derivados.

\begin{tabular}{|l|c|c|}
\hline \multicolumn{1}{|c|}{ Alimento } & \% humedad & $\begin{array}{c}\text { Na mg /l00 g de } \\
\text { alimento fresco }\end{array}$ \\
\hline $\begin{array}{l}\text { Bistec de lomo, cocido con agua desionizada en horno de } \\
\text { microondas, nacional }\end{array}$ & 62,4 & $44 \pm 2$ \\
\hline $\begin{array}{l}\text { Hígado de pollo, cocido en agua desionizada en horno de } \\
\text { microondas, sin aditivos }\end{array}$ & 68,0 & $70 \pm 3$ \\
\hline $\begin{array}{l}\text { Hígado de res, cocido con agua desionizada en horno de microondas, } \\
\text { nacional }\end{array}$ & 69,2 & $50 \pm 2$ \\
\hline $\begin{array}{l}\text { Pescado, filet de tilapia, limpio, fresco, sin escamas, cocido con agua } \\
\text { desionizada en horno de microondas, nacional }\end{array}$ & 63,5 & $44 \pm 2$ \\
\hline $\begin{array}{l}\text { Pollo, pechuga, sin piel, cocido con agua desionizada en horno de } \\
\text { microondas, nacional Pipasa }\end{array}$ & 69,8 & $44 \pm 2$ \\
\hline
\end{tabular}

Cuadro 6. Contenido de humedad y de sodio en verduras y hortalizas.

\begin{tabular}{|l|c|c|}
\hline \multicolumn{1}{|c|}{ Alimento } & \% humedad & $\begin{array}{c}\text { Na mg /l00 g de } \\
\text { alimento fresco }\end{array}$ \\
\hline Ajo, bulbo, crudo, pelado & 73,3 & $12,5 \pm 0,5$ \\
\hline $\begin{array}{l}\text { Brócoli, tallo y flor, cocido con agua desionizada en horno de } \\
\text { microondas, feria del agricultor }\end{array}$ & 91,6 & $1,8 \pm 0,1$ \\
\hline $\begin{array}{l}\text { Camote, amarillo, tubérculo, sin cáscara, cocido con agua desionizada } \\
\text { en horno de microondas, nacional, feria del agricultor }\end{array}$ & 58,3 & $2,3 \pm 0,1$ \\
\hline Cebolla, blanca, cruda, pelada & 93,2 & $15,5 \pm 0,6$ \\
\hline Chile dulce, fruto, sin semillas, crudo, nacional & 93,6 & $1,6 \pm 0,1$ \\
\hline
\end{tabular}




\begin{tabular}{|l|c|c|}
\hline $\begin{array}{l}\text { Papa, amarilla, tubérculo, sin cáscara, cocida con agua desionizada en } \\
\text { horno de microondas, nacional, feria del agricultor }\end{array}$ & 81,3 & ND \\
\hline $\begin{array}{l}\text { Plátano maduro, fruto, cocido con agua desionizada en horno de } \\
\text { microondas, nacional, feria del agricultor }\end{array}$ & 75,8 & ND \\
\hline $\begin{array}{l}\text { Zanahoria, raíz, sin cáscara, cocida con agua desionizada en horno de } \\
\text { microondas, nacional, feria del agricultor }\end{array}$ & 90,5 & $24 \pm 2$ \\
\hline
\end{tabular}

Cuadro 7. Contenido de humedad y de sodio en frutas.

\begin{tabular}{|c|c|c|}
\hline Alimento & $\%$ humedad & $\begin{array}{l}\mathrm{Na} \mathrm{mg} / / 00 \mathrm{~g} \text { de } \\
\text { alimento fresco }\end{array}$ \\
\hline Aguacate, Hass, de primera, fruto, maduro, cáscara negra verdosa & 75,9 & ND \\
\hline $\begin{array}{l}\text { Banano criollo, maduro, cáscara amarilla con manchas, fruto amarillo } \\
\text { intenso }\end{array}$ & 73,9 & ND \\
\hline $\begin{array}{l}\text { Banano, de exportación, fruto, amarillo pálido, Banasun, Premiun } \\
\text { Quality Banana }\end{array}$ & 74,2 & ND \\
\hline Fresas, maduras, rojas, fruto sin hojas, de Llano Grande de Cartago & 93,1 & ND \\
\hline Manzana Gala, cáscara rojiamarilla fruto, con cáscara, sin semilla & 85,7 & ND \\
\hline Manzana Golden, amarilla, fruto con cáscara, sin semilla & 85,7 & ND \\
\hline $\begin{array}{l}\text { Melón, Petatillo, de primera, maduro, pulpa (parte amarilla), sin } \\
\text { semillas, Sardinal de Guanacaste }\end{array}$ & 94,8 & $2,01 \pm 0,07$ \\
\hline Papaya nacional, madura, cáscara amarilla, pulpa, sin semillas & 87,6 & $1,09 \pm 0 ., 04$ \\
\hline Papaya suprema, cáscara amarilla, pulpa, sin semillas & 87,7 & $0,87 \pm 0,03$ \\
\hline Piña Hawaiana, cáscara amarillo verdoso, madura, pulpa sin corazón & 87,9 & ND \\
\hline Piña Oro, cáscara amarilla, madura, pulpa sin corazón & 83,5 & ND \\
\hline Sandía Miquiliqui, de primera, pulpa (parte roja), sin semillas & 92,1 & $0,091 \pm 0,003$ \\
\hline $\begin{array}{l}\text { Tomate, nacional, maduro, entero, con semilla, sin pelar, cáscara roja, } \\
\text { de San Isidro de Tejar }\end{array}$ & 94,3 & $1,42 \pm 0,05$ \\
\hline
\end{tabular}

\section{Agradecimientos}

Las autoras agradecen el apoyo económico brindado por la Vicerrectoría de Investigación de la Universidad de Costa Rica. A la Escuela de Química de la Universidad de Costa Rica, por el uso del laboratorio de investigación para el tratamiento de las muestras y el uso del espectrómetro de absorción atómica Varian Fast Sequential utilizado para realizar las mediciones del contenido de sodio; a la Facultad de Farmacia y al Centro de Investigación en Tecnología de Alimentos de la Universidad de Costa Rica, por facilitar el uso del liofilizador utilizado en el secado de las muestras.

\section{Bibliografía}

I. Traub, S. ed. (1992) Basic Skills in interpreting laboratory data: illustrated with case studies. 2 ed. (s.L): American Society of Health System Pharmacists, pp 94- I 15.

2. Gibney, M.j; Vorster H.H; Kok, F.J. (2005) Introducción a la nutrición humana. Zaragoza, España: Acribia, pp. 193210.
3. Wardlaw, G.M. (1993) Perspectives in Nutrition. 4 ed. New York, USA: Mc Graw Hill International, pp 472492.

4. Cutler, J.; Roccella, E. (2006). Hypertension editorial commentary: Salt reduction for preventing hypertension and cardiovascular disease - a population approach should intake should include children Hypertension 48: 818-819.

5. Brown, I. J.; Tzoulaki, I; Candeias, V.; Elliot, P. (2009) Salt intakes around the world: implications for public health. T. Int. J. Epidemiology. I-23.

6. Karppanen, H; Mervaala, E. (2006) Sodium Intake and Hypertension Progress in Cardiovascular Diseases. Volumen $49, n^{\circ} 2,59-75$.

7. Zehender, B. Sodioum, potasium and hypertension Rev. Med. Clin. CONDES - 20 I 0; 2 I (4) 508-5I5]

8. Reddy, K.S.; Katan, M.B. (2004) Diet, nutrition and the prevention of hypertension and cardiovascular diseases. Public Health Nutrition: 7( IA), 167-186

9. Ministerio de Salud. (1997) Guías alimentarias para la educación nutricional en Costa Rica. San José, Costa Rica. 
10. Inciensa. (1986) Encuesta Nacional sobre consumo de sal doméstica en el hogar, Costa Rica 1984. San José, Costa Rica.

II. Ministerio de Salud. (2003) Encuesta basal de factores de riesgo para enfermedades no transmisibles Cartago 200 I: Factores alimentario nutricionales. San José, Costa Rica, pp. 12-13.

12. WHO (World Health Organization). (2003) Diet, nutrition and the prevention of chronic diseases. Informe de una consulta mixta de expertos WHO/FAO. Ginebra, Suiza. (WHO, Serie de informes técnicos, $N^{\circ}$ 916).

13. Pacheco, P. (2007) Enfermedades cardiovasculares: Primera causa de muerte en Costa Rica. Medicina: Vida y Salud. Revista informativa oficial del Colegio de Médicos y Cirujanos de Costa Rica. Volumen 9, octubre 2007.

14. Méndez, E.; Rosero, L. (2007) Prevalencia de hipertensión en adultos mayores de Costa Rica. Población y Salud en Mesoamérica. Centro Centroamericano de Población, Universidad de Costa Rica, 5 (I).
15. Ministerio de Salud. (2006) Informe de resultados de la evaluación de la calidad de atención en hipertensión arterial en la red de servicios de salud para el año 2005. San José, Costa Rica: Ministerio de salud, Organización Panamericana de la Salud.

16. Infartos son la principal causa de muerte en Costa Rica. San José. nacion. com 2 de setiembre del 2010.

17. Scientific Advisory Committee on Nutrition. (2003) Salt and Health.TSO The Stationery Office, London.

18. World Action On Salt and Health. Introduction. Obtenido el 24 de marzo del 2011 desde www.worldactiononsalt.com

19. Cardiovascular disease prevention through dietary salt reduction: PAHO expert group. Fact sheet. Obtenido el 22 de marzo del 20II desde http://www.paho.org/ cncd_cvd/salt

20. Greenfield, H., Southgate, D. (2003). Datos de composición de alimentos. (2 a Ed. En español). Roma: FAO.

21. Official Methods of Analysis of AOAC International, Official Method 985.35, 18 th Edition, Revision \#I, 2005. 